\title{
Evaluation of Phase Effects in Geant4 Microdosimetry Models for Particle Interactions in Water
}

\author{
S. Chauvie, S. Incerti, P. Moretto, M. G. Pia
}

\begin{abstract}
This study presents an investigation of the accuracy of a selection of Physics models implemented in the Geant4 DNA extension to the Geant4 Monte Carlo toolkit and illustrates the physical effects of the interacting medium phase.
\end{abstract}

\section{INTRODUCTION}

$\mathrm{T}_{\mathrm{N}}^{\mathrm{H}}$ HE new Geant4 DNA extension [1] to the Geant4 [2],[3] Monte Carlo simulation toolkit is able to simulate interactions of electrons, protons, hydrogen, $\alpha$ particles and their charge states down to the electronVolt scale in liquid water.

This new energy régime is matter of theoretical and experimental complexity. Theoretical calculations must take into account the detailed dielectric structure of the water condensed phase; they are often based on approximations, assumptions and semi-empirical models. Experimental measurements are difficult to perform, needing a precise control of systematics, and are often limited by practical constraints depending on the interacting medium phase.

The accuracy of the Geant4 DNA physics models may be estimated from a rigorous verification and validation process of the models implementation. Verification checks the conformity of the implementation with the corresponding theoretical model and can be performed on all physics models available in Geant4 DNA. Validation is performed by comparing the models predictions against experimental data. Although a relatively large amount of experimental data exists in vapor and some in ice in the very low energy range, there is a crucial lack of experimental data in liquid water, which prevents the validation of the implemented models.

However, the plausibility of the Geant4 DNA software implementation may be investigated in comparison to available experimental data in other phases; the results may give insight into physical effects related to the phase of the interacting medium.

This study presents a selection of interesting cases for cross section modeling, highlighting the complexity of the

Manuscript received November 19, 2007.

S. Chauvie is with Azienda Ospedaliera Santa Croce e Carle Cuneo and INFN Sezione di Genova, 16146 Genova, Italy (phone: +39 0171641558 , fax: + 390171641564 , e-mail: chauvie.s@ospedale.cuneo.it).

S. Incerti and P. Moretto are with CENBG, IN2P3/CNRS, Bordeaux 1 University, F-33175 Gradignan Cedex, France (phone: +33 557120889, fax: + 33 557120801, e-mail: incerti@cenbg.in2p3.fr and moretto@cenbg.in2p3.fr).

M.G. Pia is with INFN Sezione di Genova, Via Dodecaneso 33, I-16146 Genova - Italy (phone: +39 010 3536328, fax: +39 010 313358, e-mail: MariaGrazia.Pia@ge.infn.it). experimental domain and allowing discussion of physics modeling features.

\section{ELECTRON ELASTIC SCATTERING}

Modeling electron elastic scattering at very low energy is a theoretical challenge. Various theoretical approaches at different degrees of complexity from simple screened Rutherford cross section to sophisticated phase shift calculations have been developed. The Geant4 DNA implementation of electron elastic scattering is based on the screened Rutherford cross section and a semi-empirical model based on vapor data. It is naturally open to evolution along with the availability of new data.

Unfortunately, no experimental data in liquid water are available to validate the simulation model. Comparisons against data in vapor can contribute to establish at least the plausibility of the modeling approach adopted.

The simulated total cross section of elastic scattering for electrons in liquid water is shown in Fig. 1, together with a collection of experimental data in vapor: there is an evident discrepancy of experimental data. Recommended evaluated data from Itikawa and Mason [4] show a striking inconsistency: at some energies the electron recommended total cross section is smaller than the elastic cross section.

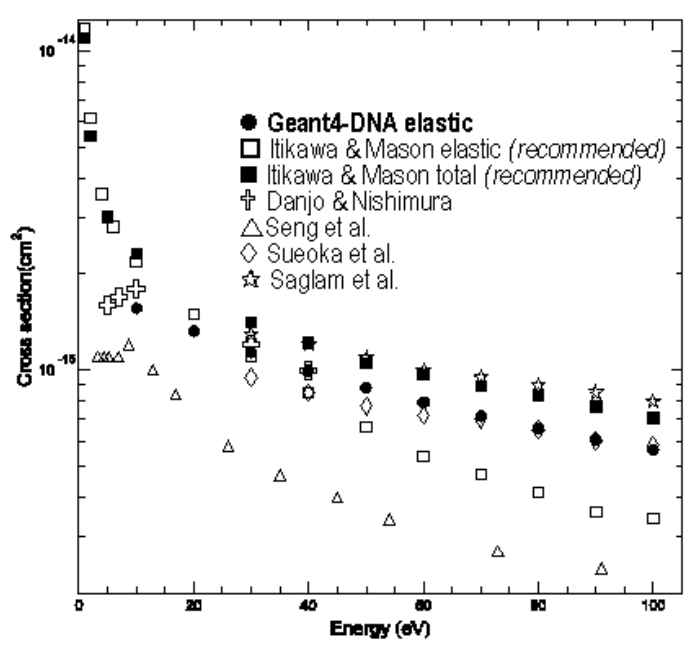

Fig. 1. Comparison of Geant4 DNA elastic scattering cross section for electrons (plain circles) versus a selection of available data [5]-[8].

\section{ELECTRON IONIZATION}

The electron ionization model uses a semi-empirical model developed by Emfietzoglou and Moscovitch [9]. It is based on 
the dielectric formalism for the valence shells $\left(1 b_{1}, 3 a_{1}, 1 b_{2}\right.$ and $2 a_{1}$ ) responsible for condensed phase effects and it is based on the binary encounter approximation for the water molecule K-shell $\left(1 a_{1}\right)$. Experimental data are found in the vapor phase.

The comparison is shown in Fig. 2, illustrating the plausible behavior of the Geant4 DNA implementation. It also suggests that phase effects may be more significant at lower energies.

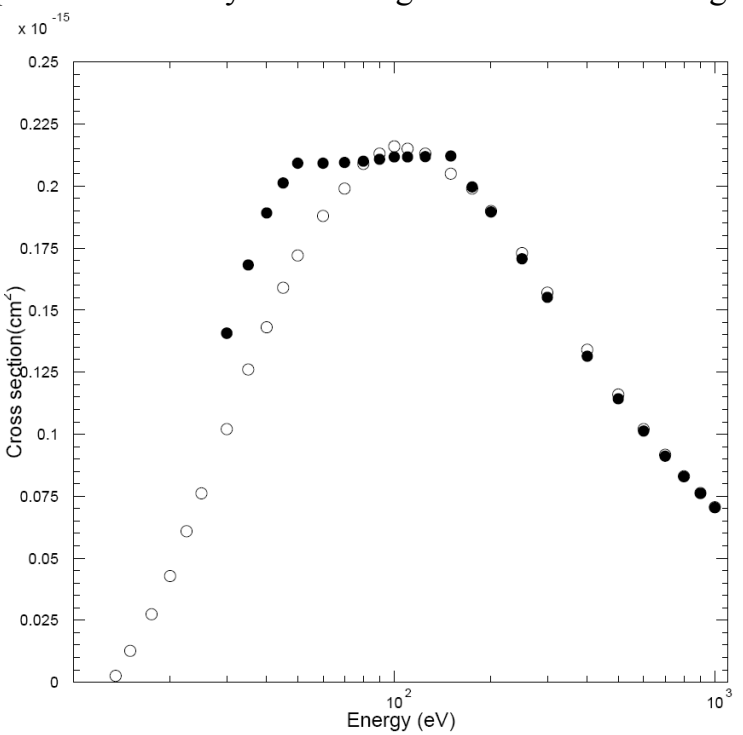

Fig. 2. Comparison of Geant4 DNA ionization cross section for electrons (plain circles) versus experimental data (open circles) [4].

\section{PRoton IONIZATION}

The cross section model is based on two complementary models. A semi-empirical analytical approach is used for lower energies in the range $100 \mathrm{eV}-500 \mathrm{keV}$ with parameters specifically calculated for liquid water. At higher energies, from $500 \mathrm{keV}$ up to $10 \mathrm{MeV}$, the modelling is based on Born theory. Experimental data are available for the vapour phase only.

Comparisons between Geant4 DNA modelling and experimental data is shown in Fig. 3. All the experimental measurements have been performed by the same team, at different accelerator facilities and times. This figure shows clearly that even data taken by the same group exhibit inconsistencies, highlighting the difficulty to control systematics in delicate experimental conditions.

The Geant4 DNA models look plausible. A statistical goodness-of-fit test can be performed on the Geant4 DNA predictions (plain line) to evaluate the compatibility of the simulation in the liquid phase against the raw experimental data in the gaseous phase and the fit (dashed line) to experimental data shown in Fig. 3. According to all the goodness-of-fit tests for unbinned distributions included in the Statistical Toolkit [10]-[11], the Geant4 DNA model is incompatible with the experimental data ( $p$-value $<0.001$ ), which anyway differ significantly against one another. The data fit is compatible with the data according to the Cramer von Mises test ( $p$-value $=0.1$ ), but it is incompatible with them according to the Anderson-Darling test ( $\mathrm{p}$-value $<0.001$ ).

\section{PRoton AND HYdRogen CHARGE CHANGE}

The cross section model is based on a semi-empirical approach described by an analytical formula with parameters optimized from experimental data in vapour. They are shown in Fig. 4. Experimental data are available in this phase only. Goodness-of-fit tests may be performed between the Geant 4 DNA predictions and the experimental data (keeping only data displayed with white symbols in Fig. 4). Anderson-Darling, Cramer-von Mises, Kolmogorov-Smirnov, Kuiper and Watson tests give all $\mathrm{p}$-values $>0.1$. However, this nice agreement should not hide that some of these data were used to optimize the semi-empirical model.

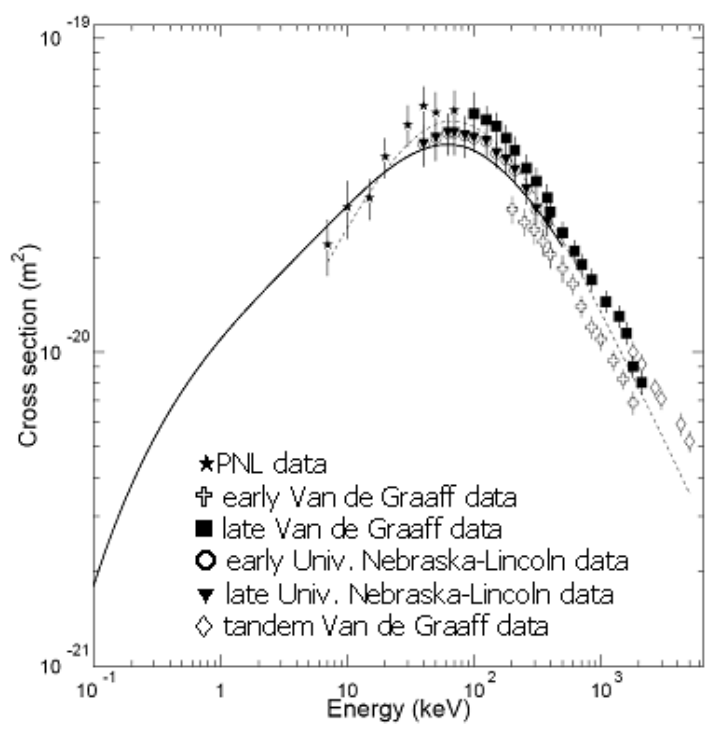

Fig. 3. Comparison of Geant4 DNA ionization cross section for protons (plain line) versus experimental data [12]. A fit to the experimental data is shown (dashed line).

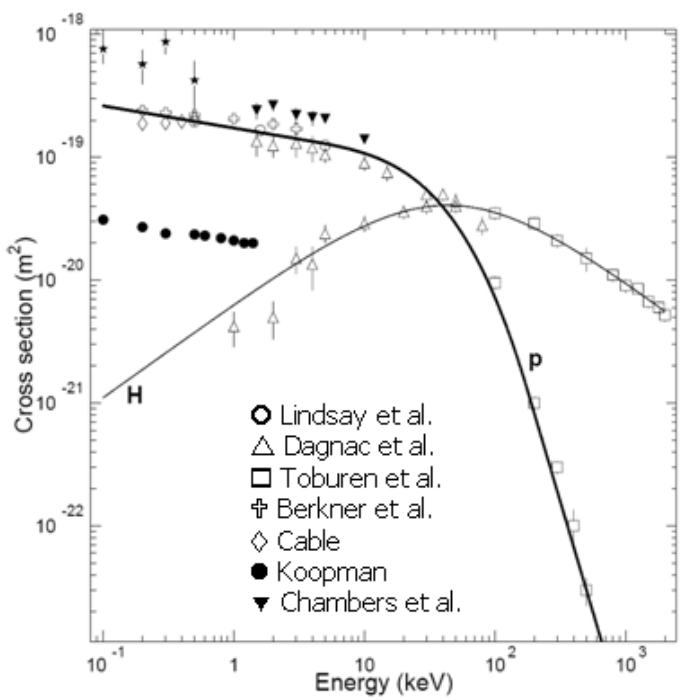

Fig. 4. Comparison of Geant4 DNA charge change cross section for hydrogen (thin line) and protons (thick line) versus experimental data: [13] (white circles), [14] (white triangles), [15] (squares), [16] (crosses), the Cable data series (diamonds) and the the Koopman one (black circles) reported in [17], [18] (black triangles), and [19] (stars) 


\section{CONCLUSION}

This study shows that Geant4 DNA physics models, which are valid for the water liquid phase only, look plausible when they are compared to available experimental data, mainly in the vapor phase.

More experimental data are obviously needed, not only in the liquid phase to validate further these models, but also in the vapor and ice phases to investigate the importance of phase effects in modeling particle interactions with the water medium.

It is hard to draw firm conclusions about phase effects at this stage. The available experimental data in vapor exhibit significant discrepancies in many cases and some of these data have already been used to constrain or optimize semiempirical models. A systematic comparison will be performed from a more extensive collection of published data and will be published in a dedicated paper.

\section{REFERENCES}

[1] S. Chauvie, Z. Francis, S. Guatelli, S. Incerti, B. Mascialino, P. Moretto, P. Nieminen and M. G. Pia, "Geant4 physics processes for microdosimetry simulation: design foundation and implementation of the first set of models", to be published in IEEE Trans. Nucl. Sci., Dec. 2007

[2] S. Agostinelli et al., "Geant4 - a Simulation Toolkit", Nucl. Instrum. Methods A, vol. 506 pp. 250-303, Jul. 2003.

[3] J. Allison, K. Amako, J. Apostolakis, H. Araujo, P. Arce Dubois, M. Asai, et al., "Geant4 developments and applications", IEEE Trans. Nucl. Sci., vol. 53, no. 1, pp. 270-278, Feb. 2006.

[4] Itikawa and N. Mason, "Cross sections for electron collisions with water molecules", J. Phys. Chem. Ref. Data, Vol. 34, No. 1, 2005, pp. 1-22

[5] A. Danjo and H. Nishimura, "Elastic Scattering of Electrons from $\mathrm{H}_{2} \mathrm{O}$ Molecule", J. Phys. Soc. of Japan, nol. 54 no. 4, pp. 1224-1227, 1985.

[6] Z. Saglam and N. Aktekin, "Absolute total cross section for electron scattering on water in the energy range 25-300 eV", J. Phys. B: At. Mol. Opt. Phys., vol. 23, 1529-1536, 1990.

[7] G. Seng, "Das system e- $\mathrm{H}_{2} \mathrm{O}$ : Differentielle Streuexperiment bei kleinsten Energien“, Thesis, Kaiserslautern Univ., 1975.

[8] O. Sueoka, S. Mori and Y. Katayama, "Total cross sections for electrons and positrons colliding with $\mathrm{H} 2 \mathrm{O}$ molecules", J. Phys. B: At. Mol. Phys. 19, pp. L373-L378, 1986.

[9] D. Emfietzoglou and M. Moscovitch, "Inelastic collision characteristics of electrons in liquid water", NIM B, vol. 193, pp. 71-78, 2002

[10] G. A. P. Cirrone et al., "A Goodness-of-Fit Statistical Toolkit", IEEE Trans. Nucl. Sci., vol. 51, no. 5, pp. 2056-2063, Oct. 2004.

[11] B. Mascialino, A. Pfeiffer, M. G. Pia, A. Ribon, and P. Viarengo, "New developments of the Goodness-of-Fit Statistical Toolkit", IEEE Trans. Nucl. Sci., vol. 53, no. 6, pp. 3834-3841, Dec. 2006.

[12] M. E. Rudd et al., "Cross sections for ionisation of water vapor by 7 $4000 \mathrm{keV}$ protons", Phys. Rev. A, vol. 31, pp. 492-494, 1985.

[13] B. G. Lindsay et al., "Charge transfer of $0.5-, 1.5-$, and $5-\mathrm{keV}$ protons with H2O: absolute differential and integral cross sections", Phys. Rev. A, vol. 55, no. 5, pp. 3945-3946, 1997.

[14] R. Dagnac et al., "A study on the collision of hydrogen ions $\mathrm{H}+1, \mathrm{H}+2$ and $\mathrm{H}+3$ with a water-vapour target", J. Phys. B, vol. 3, pp. 1239-1251, 1970.

[15] L. H. Toburen et al., "Measurement of highenergy charge transfer cross sections for incident protons and atomic hydrogen in various gases", Phys. Rev., vol. 171, no. 1, pp. 114-122, 1968

[16] K. H. Berkner et al., "Cross sections for electron capture by 0.3 to 70 $\mathrm{keV}$ deuterons in $\mathrm{H} 2, \mathrm{H} 2 \mathrm{O}, \mathrm{CO}, \mathrm{CH} 4$, and $\mathrm{C} 8 \mathrm{~F} 16$-gases", Nucl. Fus., vol. 10, pp. 145-149, 1970

[17] D. W. Koopman, "Light-ion charge exchange in atmospheric gases", Phys. Rev., vol. 166, pp. 57-62, 1968.

[18] E. S. Chambers, Lawrence Radiation Laboratory Report UCRL-14214, 1965.

[19] M. A. Coplan and K. W. Ogilvie, "Charge exchange for $\mathrm{H}+$-and $\mathrm{H}+2$ in H2O, CO2, and NH3”, J. Chem. Phys., vol. 52, pp. 4154-4160, 1970. 